\title{
RAMANUJAN'S SCHLÄFLI-TYPE MODULAR EQUATIONS AND CLASS INVARIANTS $g_{n}$
}

NIPEN SAIKIA

Abstract: In this paper, we use Ramanujan's Schläfli-type modular equations to find some new values of class invariants $g_{n}$ and also give alternate proofs of some of known values.

Keywords: modular equation, class invariant.

\section{Introduction}

The Dedekind eta-function $\eta(z)$ is defined by

$$
\eta(z)=e^{\pi i z / 12} \prod_{n=1}^{\infty}\left(1-e^{2 \pi i n z}\right), \quad \operatorname{Im}(z)>0 .
$$

Following Ramanujan's notation, we set $q:=e^{2 \pi i z}$ and

$$
f(-q)=(q ; q)_{\infty}=q^{-1 / 24} \eta(z),
$$

where $(a ; q)_{\infty}:=\prod_{k=1}^{\infty}\left(1-a q^{k-1}\right)$.

Now, for $q:=e^{-\pi \sqrt{n}}$, where $n$ is a positive rational number, Weber-Ramanujan class invariants $G_{n}$ and $g_{n}[5$, p. 183, (1.3)] are defined by

$$
G_{n}=2^{-1 / 4} q^{-1 / 24} \chi(q) \quad \text { and } \quad g_{n}=2^{-1 / 4} q^{-1 / 24} \chi(-q),
$$

where $\chi(q)=\left(-q ; q^{2}\right)_{\infty}$.

Since from [3, p. 124], $\chi(q)=2^{1 / 6}\{\alpha(1-\alpha) / q\}^{-1 / 24}$ and $\chi(-q)=2^{1 / 6}(1-$ $\alpha)^{1 / 12}(\alpha / q)^{-1 / 24}$, it follows from (1.3) that

$$
G_{n}=\{4 \alpha(1-\alpha)\}^{-1 / 24} \quad \text { and } \quad g_{n}=2^{-1 / 12}(1-\alpha)^{1 / 12} \alpha^{-1 / 24} .
$$

The author is thankful to University Grants Commission, New Delhi, India for partially supporting the research work under the grant no. F. No. 41-1394/2012(SR).

2010 Mathematics Subject Classification: primary: 33D90; secondary: 11F20 
Also, if $\beta$ has degree $r$ over $\alpha$, then

$$
G_{r^{2} n}=\{4 \beta(1-\beta)\}^{-1 / 24} \quad \text { and } \quad g_{r^{2} n}=2^{-1 / 12}(1-\beta)^{1 / 12} \beta^{-1 / 24} .
$$

In his paper [6] and notebooks [7], Ramanujan recorded a total of 116 class invariants. An account of Ramanujan's class invariants and applications can be found in Berndt's book [5].

In 2001, Yi [10] evaluated several class invariants $g_{n}$ by finding explicit values of her parameter $r_{k, n}[10$, p. 11, (2.1.1)](also see [11, p. 4, (1.11)]), defined by

$$
r_{k, n}:=\frac{f(-q)}{k^{1 / 4} q^{(k-1) / 24} f\left(-q^{k}\right)} ; \quad q=e^{-2 \pi \sqrt{n / k}},
$$

where $n$ and $k$ are positive real numbers. In particular, she established the result [10, p. 18, Theorem 2.2.3]

$$
g_{n}=r_{2, n / 2} \text {. }
$$

From [10, p. 12, Theorem 2.1.2(i)-(iii)]), we also note that

$$
r_{k, 1}=1, \quad r_{k, 1 / n}=1 / r_{k, n} \quad \text { and } \quad r_{k, n}=r_{n, k} .
$$

More recently, Saikia [8] evaluated several new values of $g_{n}$ and also proved some known values of $G_{n}$ by using Ramanujan's modular equations of prime degree. Saikia [9] also evaluated some new values of class invariant $G_{n}$.

Baruah [1] used Ramanujan's Schläfli-type modular equations of composite degrees combined with the prime degrees to prove some values of Ramanujan's class invariants $G_{n}$ but no value of $g_{n}$ is evaluated. In this paper, we show that same modular equations can also be used to find some new values of class invariants $g_{n}$. In the process, we also give alternate proofs of some of known values of class invariants $g_{n}$.

Since modular equations are key in our proofs, we now define a modular equation. Let $K, K^{\prime}, L$, and $L^{\prime}$ denote the complete elliptic integrals of the first kind associated with the moduli $k, k^{\prime}, l$, and $l^{\prime}$, respectively. Suppose that the equality

$$
n \frac{K^{\prime}}{K}=\frac{L^{\prime}}{L}
$$

holds for some positive integer $n$. Then a modular equation of degree $n$ is a relation between the moduli $k$ and $l$ which is implied by (1.9). Ramanujan recorded his modular equations in terms of $\alpha$ and $\beta$ where $\alpha=k^{2}$ and $\beta=l^{2}$. We say that $\beta$ has degree $n$ over $\alpha$. The multiplier $m$ connecting $\alpha$ and $\beta$ is defined by

$$
m=\frac{K}{L},
$$

where $z_{r}=\phi^{2}(q)$. Similarly, one can define Ramanujan's " mixed "modular equation or modular equation of composite degree. We refer to Chapter 20 of Berndt's book [3]. 
In Section 2, we list some Schläfli-type modular equations which will be used in the subsequent sections. In Section 3, we evaluate some new as well as some known values of the class invariant $g_{n}$.

We end this introduction by recalling from [3, p. 124, Entry 12(i), (iii)], that

$$
f(q)=\sqrt{z} 2^{-1 / 6}(\alpha(1-\alpha) q)^{1 / 24} \quad \text { and } \quad f\left(-q^{2}\right)=\sqrt{z} 2^{-1 / 3}(\alpha(1-\alpha) q)^{1 / 12}
$$

\section{Schläfli-type modular equations}

This section is devoted to recording some Schläfli-type modular equations. In the first three lemmas, we set

$$
L:=2^{1 / 6}(\alpha \beta(1-\alpha)(1-\beta))^{1 / 24} \quad \text { and } \quad S:=\left(\frac{\beta(1-\beta)}{\alpha(1-\alpha)}\right)^{1 / 24} .
$$

Lemma 2.1 ([5, p. 378, Entry 41]). If $\beta$ has degree 11 over $\alpha$, then

$$
S^{6}+\frac{1}{S^{6}}-2 \sqrt{2}\left(\frac{2}{L^{5}}-\frac{11}{L^{3}}+\frac{22}{L}-22 L+11 L^{3}-2 L^{5}\right)=0 .
$$

Lemma 2.2 ([5, p. 378, Entry 41]). If $\beta$ has degree 13 over $\alpha$, then

$$
S^{7}+\frac{1}{S^{7}}+13\left(S^{5}+\frac{1}{S^{5}}\right)+52\left(S^{3}+\frac{1}{S^{3}}\right)+78\left(S+\frac{1}{S}\right)-8\left(L^{6}-\frac{1}{L^{6}}\right)=0
$$

Lemma 2.3 ([5, p. 378, Entry 41]). If $\beta$ has degree 17 over $\alpha$, then

$$
\begin{aligned}
S^{9}+\frac{1}{S^{9}}-34\left(S^{6}+\frac{1}{S^{6}}\right)+ & 17\left(S^{3}+\frac{1}{S^{3}}\right)\left(\frac{4}{L^{4}}+7+4 L^{4}\right) \\
- & \left(\frac{16}{L^{8}}-\frac{136}{L^{4}}-340-136 L^{4}+16 L^{8}\right)=0 .
\end{aligned}
$$

In the remaining lemmas of this section, we set

$$
\begin{aligned}
P & :=(256 \alpha \beta \gamma \delta(1-\alpha)(1-\beta)(1-\gamma)(1-\delta))^{1 / 48}, \\
Q & :=\left(\frac{\alpha \delta(1-\alpha)(1-\delta)}{\beta \gamma(1-\beta)(1-\gamma)}\right)^{1 / 48}, \\
R & :=\left(\frac{\gamma \delta(1-\gamma)(1-\delta)}{\alpha \beta(1-\alpha)(1-\beta)}\right)^{1 / 48},
\end{aligned}
$$

and

$$
T:=\left(\frac{\beta \delta(1-\beta)(1-\delta)}{\alpha \gamma(1-\alpha)(1-\gamma)}\right)^{1 / 48}
$$


Lemma 2.4 ([5, p. 381, Entry 50]). If $\alpha, \beta, \gamma$, and $\delta$ have degrees 1, 5, 7, and 35, respectively, then

$$
R^{4}+\frac{1}{R^{4}}-\left(Q^{6}+\frac{1}{Q^{6}}\right)+5\left(Q^{4}+\frac{1}{Q^{4}}\right)-10\left(Q^{2}+\frac{1}{Q^{2}}\right)+15=0 .
$$

Lemma 2.5 ([5, p. 381, Entry 48]). If $\alpha, \beta, \gamma$, and $\delta$ have degrees 5, 1, 7 , and 35 , respectively, then

$$
Q^{6}+\frac{1}{Q^{6}}+5 \sqrt{2}\left(Q^{3}+\frac{1}{Q^{3}}\right)\left(P+\frac{1}{P}\right)-4\left(P^{4}+\frac{1}{P^{4}}\right)+10=0 .
$$

Lemma 2.6 ([5, p. 380, Entry 43]). If $\alpha, \beta, \gamma$, and $\delta$ have degrees 3, 1, 5, and 15 , respectively, then

$$
Q^{4}+\frac{1}{Q^{4}}-2\left(P^{2}+\frac{1}{P^{2}}\right)+3=0
$$

Lemma 2.7 ([5, p. 381, Entry 51]). If $\alpha, \beta, \gamma$, and $\delta$ have degrees 1, 13, 3, and 39, respectively, then

$$
Q^{4}+\frac{1}{Q^{4}}-3\left(Q^{2}+\frac{1}{Q^{2}}\right)-\left(T^{2}+\frac{1}{T^{2}}\right)+3=0
$$

Lemma 2.8 ([5, p. 380, Entry 47]). If $\alpha, \beta, \gamma$, and $\delta$ have degrees 3, 1, 11, and 33, respectively, then

$$
Q^{4}+\frac{1}{Q^{4}}+3\left(Q^{2}+\frac{1}{Q^{2}}\right)-2\left(P^{2}+\frac{1}{P^{2}}\right)=0 .
$$

Lemma 2.9 ([5, p. 380, Entry 44]). If $\alpha, \beta, \gamma$, and $\delta$ have degrees 5, 1, 3, and 15 , respectively, then

$$
Q^{6}+\frac{1}{Q^{6}}-4\left(P^{4}+\frac{1}{P^{4}}\right)+10\left(P^{2}+\frac{1}{P^{2}}-1\right)=0 .
$$

Lemma 2.10 ([2, p. 277, Lemma 3.1]). If $\alpha, \beta, \gamma$, and $\delta$ have degrees 1, 3, 7 , and 21 , respectively, then

$$
R^{2}+\frac{1}{R^{2}}=Q^{4}+\frac{1}{Q^{4}}-3
$$

Lemma 2.11 ([2, p. 283, Theorem 4.1]). If $\alpha, \beta, \gamma$, and $\delta$ have degrees 1, 3, 7 , and 21, respectively, then

$$
\begin{aligned}
T^{12}+\frac{1}{T^{12}}-18\left(T^{6}+\frac{1}{T^{6}}\right)+18 \sqrt{2}\left(T^{3}+\frac{1}{T^{3}}\right) & \left(P^{3}+\frac{1}{P^{3}}\right) \\
& -8\left(P^{6}+\frac{1}{P^{6}}\right)-54=0 .
\end{aligned}
$$




\section{Values of $g_{n}$}

In this section, we find some values of $g_{n}$ by using the Schläfli-type modular equations recorded in the previous section.

Theorem 3.1. We have

$$
g_{22}=(19601+13860 \sqrt{2})^{1 / 24} \quad \text { and } \quad g_{2 / 11}=(19601-13860 \sqrt{2})^{1 / 24} .
$$

The value $g_{22}$ can also be found in [5, p. 200].

Proof. We set

$$
A:=\frac{f(q)}{q^{1 / 24} f\left(-q^{2}\right)} \quad \text { and } \quad B:=\frac{f\left(q^{11}\right)}{q^{11 / 24} f\left(-q^{22}\right)} .
$$

so that, by (1.11), we have

$$
A=\frac{2^{1 / 6}}{(\alpha(1-\alpha))^{1 / 24}} \quad \text { and } \quad B=\frac{2^{1 / 6}}{(\beta(1-\beta))^{1 / 24}},
$$

where $\beta$ has degree 11 over $\alpha$.

Now, from (2.1) and (3.2), we find that

$$
L=2^{1 / 2} /(A B) \quad \text { and } \quad S=A / B .
$$

Replacing $q$ by $-q$, we observe that $L^{2}$ and $S^{12}$ are transformed into $-L_{1}^{2}$ and $-S_{1}^{12}$, respectively, where

$$
L_{1}=2^{1 / 2} /\left(A_{1} B_{1}\right) \quad \text { and } \quad S_{1}=A_{1} / B_{1},
$$

where

$$
A_{1}=\frac{f(-q)}{q^{1 / 24} f\left(-q^{2}\right)}, \quad \text { and } \quad B_{1}=\frac{f\left(-q^{11}\right)}{q^{11 / 24} f\left(-q^{22}\right)} .
$$

Consequently, Lemma 2.1 gives

$$
\begin{aligned}
9746+\frac{32}{L_{1}^{10}}+\frac{352}{L_{1}^{8}}+\frac{1672}{L_{1}^{6}} & +\frac{4576}{L_{1}^{4}}+\frac{8096}{L_{1}^{2}}+8096 L_{1}^{2}+4576 L_{1}^{4} \\
& +1672 L_{1}^{6}+352 L_{1}^{8}+32 L_{1}^{12}-\frac{1}{S_{1}^{12}}-S_{1}^{12}=0
\end{aligned}
$$

Now, setting $q=e^{-2 \pi \sqrt{n / 2}}$ and applying the definition of $r_{k, n}$, for $k=2$, in (3.4), we obtain

$$
L_{1}=\left(r_{2, n} r_{2,121 n}\right)^{-1} \quad \text { and } \quad S_{1}=\left(r_{2, n} / r_{2,121 n}\right) .
$$

Setting $n=1 / 11$ in (3.7) and using (1.8), we find that

$$
L_{1}=1 \quad \text { and } \quad S_{1}=r_{2,11}^{-2} .
$$


Invoking (3.8) in (3.6) and simplifying, we find that

$$
r_{2,11}^{24}+r_{2,11}^{-24}-39202=0 .
$$

Solving (3.9) for real positive value of $r_{2,11}>1$, we obtain

$$
r_{2,11}=(19601+13860 \sqrt{2})^{1 / 24} .
$$

Using (1.7) and (1.8), we complete the proof.

The proofs of Theorems 3.2 and 3.4 are identical to the proof of Theorem 3.1, so we skip details of the proofs.

Theorem 3.2. We have

$$
g_{26}=\left(\frac{1}{6}\left(m+\sqrt{-36+m^{2}}\right)\right)^{1 / 4} \text { and } g_{2 / 13}=\left(\frac{1}{6}\left(m-\sqrt{-36+m^{2}}\right)\right)^{1 / 4} \text {, }
$$

where

$$
m=8+(359-12 \sqrt{78})^{1 / 3}+(359+12 \sqrt{78})^{1 / 3} .
$$

The value $g_{26}$ can also be found in [5, p. 200].

Proof. Proceeding as in the proof of Theorem 3.1, expressing Lemma 2.2 in etafunction $f$ and then replacing $q$ by $-q$, we use the definition of $r_{2, n}$, set $n=1 / 13$ and employ (1.8) to arrive at

$$
\left(1-9 x^{4}+20 x^{8}-9 x^{12}+x^{16}\right)^{2}\left(1-8 x^{4}+8 x^{8}-18 x^{12}+8 x^{16}-8 x^{20}+x^{24}\right)=0,
$$

where $x=r_{2,13}$.

Since the first two equal factors have no real root for $r_{2,13}$, we arrive at

$$
1-8 r_{2,13}^{4}+8 r_{2,13}^{8}-18 r_{2,13}^{12}+8 r_{2,13}^{16}-8 r_{2,13}^{20}+r_{2,13}^{24}=0 .
$$

Setting $z=r_{2,13}^{4}+r_{2,13}^{-4}$ in the (3.12), we find that

$$
z^{3}-8 z^{2}+5 z-2=0
$$

Solving (3.13) for real positive value of $z>1$, we have

$$
z=\left(8+(359-12 \sqrt{78})^{1 / 3}+(359+12 \sqrt{78})^{1 / 3}\right) / 3,
$$

and hence,

$$
r_{2,13}^{4}=\left(m+\sqrt{-36+m^{2}}\right) / 6,
$$

where $m=8+(359-12 \sqrt{78})^{1 / 3}+(359+12 \sqrt{78})^{1 / 3}$. Now employing (1.7) and (1.8) in (3.15) and simplifying, we complete the proof. 
Remark 3.3. The values of $g_{26}$ and $g_{2 / 13}$ can also be obtained by using the eta-function identity [4, p. 211, Entry 57] instead of Lemma 2.2.

Theorem 3.4. We have

$$
g_{34}=(9+2 \sqrt{17}+2 \sqrt{37+9 \sqrt{17}})^{1 / 6}
$$

and

$$
g_{2 / 17}=(9+2 \sqrt{17}-2 \sqrt{37+9 \sqrt{17}})^{1 / 6} .
$$

The value $g_{34}$ can also be found in [5, p. 200].

Proof. Expressing Lemma 2.3 in $f$, replacing $q$ by $-q$ and then employing the definition $r_{2, n}$ and (1.8) for $n=1 / 17$, we deduce that

$$
r_{2,17}^{18}+r_{2,17}^{-18}-17\left(r_{2,17}^{6}+r_{2,17}^{-6}\right)-34\left(r_{2,17}^{12}+r_{2,17}^{-12}\right)+36=0 .
$$

Solving the above equation for real positive $r_{2,17}$, we obtain

$$
r_{2,17}=(9+2 \sqrt{17}+\sqrt{37+9 \sqrt{17}})^{1 / 6} .
$$

Employing (1.7) and (1.8) in (3.17) we complete the proof.

Remark 3.5. Similarly, by applying the definition of $r_{2, n}$ in Lemma 2.3, setting $n=1$ and noting $r_{2,1}=1$ from (1.8), the values of $g_{578}$ and $g_{2 / 289}$ can be obtained.

Theorem 3.6. We have

$$
\begin{aligned}
g_{10 / 7} & =\left(\frac{47-21 \sqrt{5}}{2}\right)^{1 / 8}(99+70 \sqrt{2})^{1 / 12}, \\
g_{14 / 5} & =\left(\frac{47+21 \sqrt{5}}{2}\right)^{1 / 8}(99-70 \sqrt{2})^{1 / 12}, \\
g_{70} & =\left(\frac{47+21 \sqrt{5}}{2}\right)^{1 / 8}(99+70 \sqrt{2})^{1 / 12}, \\
g_{2 / 35} & =\left(\frac{47-21 \sqrt{5}}{2}\right)^{1 / 8}(99-70 \sqrt{2})^{1 / 12} .
\end{aligned}
$$

The value $g_{70}$ can also be found in [5, p. 201].

Proof. We set

$$
\begin{array}{ll}
A:=\frac{f(q)}{q^{1 / 24} f\left(-q^{2}\right)}, & B:=\frac{f\left(q^{5}\right)}{q^{5 / 24} f\left(-q^{10}\right)} \\
C:=\frac{f\left(q^{7}\right)}{q^{7 / 24} f\left(-q^{14}\right)}, & D:=\frac{f\left(q^{35}\right)}{q^{35 / 24} f\left(-q^{70}\right)}
\end{array}
$$


Transforming (3.18) by using (1.11), we get

$$
A=\frac{2^{1 / 6}}{(\alpha(1-\alpha))^{1 / 24}}, \quad B=\frac{2^{1 / 6}}{(\beta(1-\beta))^{1 / 24}}, \quad C=\frac{2^{1 / 6}}{(\gamma(1-\gamma))^{1 / 24}},
$$

and

$$
D=\frac{2^{1 / 6}}{(\delta(1-\delta))^{1 / 24}}
$$

where $\alpha, \beta, \gamma$, and $\delta$ have degrees $1,5,7$, and 35, respectively. Thus, from (2.6), (2.7), and (3.19) we find that

$$
Q^{2}=\frac{B C}{A D} \quad \text { and } \quad R^{2}=\frac{A B}{C D} .
$$

Replacing $q$ by $-q$, we observe that $Q^{2}$ and $R^{4}$ changed into $-Q_{1}^{2}$ and $-R_{1}^{4}$, respectively, such that

$$
Q_{1}^{2}=\frac{B_{1} C_{1}}{A_{1} D_{1}} \quad \text { and } \quad R_{1}^{2}=\frac{A_{1} B_{1}}{C_{1} D_{1}}
$$

where

$$
\begin{aligned}
A_{1} & =\frac{f(-q)}{q^{1 / 24} f\left(-q^{2}\right)}, & B_{1} & =\frac{f\left(-q^{5}\right)}{q^{5 / 24} f\left(-q^{10}\right)}, \\
C_{1} & =\frac{f\left(-q^{7}\right)}{q^{7 / 24} f\left(-q^{14}\right)}, & D_{1} & =\frac{f\left(-q^{35}\right)}{q^{35 / 24} f\left(-q^{70}\right)} .
\end{aligned}
$$

Consequently, Lemma 2.4 is transformed into

$$
R_{1}^{4}+\frac{1}{R_{1}^{4}}-\left(Q_{1}^{6}+\frac{1}{Q_{1}^{6}}\right)-5\left(Q_{1}^{4}+\frac{1}{Q_{1}^{4}}\right)-10\left(Q_{1}^{2}+\frac{1}{Q_{1}^{2}}\right)-15=0
$$

Setting $q:=e^{-2 \pi \sqrt{n / 2}}$ and applying the definition of $r_{k, n}$, for $k=2$, in (3.21) and (3.22), we find that

$$
Q_{1}^{2}=\frac{r_{2,25 n} r_{2,49 n}}{r_{2, n} r_{2,1225 n}} \quad \text { and } \quad R_{1}^{2}=\frac{r_{2, n} r_{2,25 n}}{r_{2,49 n} r_{2,1225 n}}
$$

Setting $n=1 / 35$ in (3.24) and using (1.8), we obtain

$$
Q_{1}^{2}=1 \quad \text { and } \quad R_{1}^{2}=\left(\frac{r_{2,5 / 7}}{r_{2,35}}\right)^{2} .
$$

Invoking (3.25) in (3.23), we deduce that

$$
\left(\frac{r_{2,5 / 7}}{r_{2,35}}\right)^{4}+\left(\frac{r_{2,5 / 7}}{r_{2,35}}\right)^{-4}-47=0
$$


Solving (3.26) for positive real value of $\left(r_{2,5 / 7} / r_{2,35}\right)$, we obtain

$$
\left(\frac{r_{2,5 / 7}}{r_{2,35}}\right)=\left(\frac{47-21 \sqrt{5}}{2}\right)^{1 / 4}
$$

As above, expressing Lemma 2.5 in $f$, replacing $q$ and $-q$ and then applying the definition to $r_{2, n}$, setting $n=1 / 35$ and employing (1.8), we deduce that

$$
\left(r_{2,5 / 7} r_{2,35}\right)^{6}+\left(r_{2,5 / 7} r_{2,35}\right)^{-6}-198=0 .
$$

Solving the (3.28) for positive real value $\left(r_{2,5 / 7} r_{2,35}\right)$, we obtain

$$
\left(r_{2,5 / 7} r_{2,35}\right)=(99+70 \sqrt{2})^{1 / 6}
$$

With the help of (3.27), (3.29), (1.7) and (1.8), the values of $g_{10 / 7}, g_{14 / 5}, g_{70}$, and $g_{2 / 35}$ readily follow.

Proofs of the Theorems 3.6, 3.7, and 3.8 being identical, so for brevity details of the proofs are omitted in next two theorems.

Theorem 3.7. We have

$$
\begin{aligned}
g_{10 / 3} & =\left(\frac{7-3 \sqrt{5}}{2}\right)^{1 / 8}(19+6 \sqrt{10})^{1 / 12} \\
g_{6 / 5} & =\left(\frac{7+3 \sqrt{5}}{2}\right)^{1 / 8}(19-6 \sqrt{10})^{1 / 12} \\
g_{30} & =\left(\frac{7+3 \sqrt{5}}{2}\right)^{1 / 8}(19+6 \sqrt{10})^{1 / 12} \\
g_{2 / 15} & =\left(\frac{7-3 \sqrt{5}}{2}\right)^{1 / 8}(19-6 \sqrt{10})^{1 / 12} .
\end{aligned}
$$

The value $g_{30}$ can also be found in [5, p. 201].

Proof. Transforming Lemma 2.6 in eta-function $f$ and then replacing the $q$ by $-q$, we employ the definition of $r_{2, n}$, set $n=1 / 15$ and use (1.8) to arrive at

$$
\left(r_{2,5 / 3} / r_{2,15}\right)^{4}+\left(r_{2,5 / 3} / r_{2,15}\right)^{-4}-7=0 .
$$

Solving the (3.30) for positive real value of $\left(r_{2,5 / 3} / r_{2,15}\right)$, we obtain

$$
\left(r_{2,5 / 3} / r_{2,15}\right)=\left(\frac{7-3 \sqrt{5}}{2}\right)^{1 / 4} .
$$


Similarly, employing the definition of $r_{2, n}$ in Lemma 2.9, setting $n=1 / 15$ and invoking (1.8), we deduce that

$$
\left(r_{2,5 / 3} r_{2,15}\right)^{6}+\left(r_{2,5 / 3} r_{2,15}\right)^{-6}-38=0
$$

Solving (3.32) for positive real value of $\left(r_{2,5 / 3} r_{2,15}\right)$, we arrive at

$$
\left(r_{2,5 / 3} r_{2,15}\right)=(19+6 \sqrt{10})^{1 / 6}
$$

The values of $g_{10 / 3}, g_{6 / 5}, g_{30}$, and $g_{2 / 15}$ now follow from $(1.7),(1.8),(3.31)$ and (3.33).

Theorem 3.8. We have

$$
\begin{aligned}
g_{6 / 7} & =\left(\frac{5-\sqrt{21}}{2}\right)^{1 / 4}(15+4 \sqrt{14})^{1 / 12} \\
g_{14 / 3} & =\left(\frac{5+\sqrt{21}}{2}\right)^{1 / 4}(15-4 \sqrt{14})^{1 / 12} \\
g_{42} & =\left(\frac{5+\sqrt{21}}{2}\right)^{1 / 4}(15+4 \sqrt{14})^{1 / 12} \\
g_{2 / 21} & =\left(\frac{5-\sqrt{21}}{2}\right)^{1 / 4}(15-4 \sqrt{14})^{1 / 12} .
\end{aligned}
$$

The value $g_{42}$ can also be found in [5, p. 201].

Proof. Transforming Lemma 2.10 in function $f$, replace $q$ by $-q$ and then applying the definition of $r_{2, n}$, setting $n=1 / 21$ and employing (1.8), we obtain

$$
\left(r_{2,3 / 7} / r_{2,21}\right)^{2}+\left(r_{2,3 / 7} / r_{2,21}\right)^{-2}-5=0 .
$$

Solving the (3.34) for real positive value of $\left(r_{2,3 / 7} / r_{2,21}\right)$, we obtain

$$
\left(r_{2,3 / 7} / r_{2,21}\right)=\left(\frac{5-\sqrt{21}}{2}\right)^{1 / 2}
$$

Again, after routine work, we apply the definition of $r_{2, n}$ in Lemma 2.11 and employing (1.8) with $n=1 / 21$, we have

$$
\left\{x^{12}+\frac{1}{x^{12}}-18\left(x^{6}+\frac{1}{x^{6}}\right)-70\right\}^{2}=2592\left(x^{6}+\frac{1}{x^{6}}\right)+5148
$$

where $x=\left(r_{2,3 / 7} r_{2,21}\right)$. 
Solving (3.36) for $x$ and noticing that $r_{2, n}>r_{2, m}$ for $n>m$, we deduce that

$$
x:=\left(r_{2,3 / 7} r_{2,21}\right)=(15+4 \sqrt{14})^{1 / 6} \text {. }
$$

The values of $g_{6 / 7}, g_{14 / 3}, g_{42}$, and $g_{2 / 21}$ follow from $(3.35),(3.37)$ and the properties (1.7) and (1.8).

Theorem 3.9. We have

$$
\begin{aligned}
g_{22 / 3}= & \frac{1}{2}(3+\sqrt{33}-\sqrt{26+6 \sqrt{33}})^{1 / 2}(\sqrt{2}+\sqrt{3})^{1 / 4} \\
& \times(7 \sqrt{2}+3 \sqrt{11})^{1 / 12}\left(\sqrt{\frac{7+\sqrt{33}}{8}}+\sqrt{\frac{\sqrt{33}-1}{8}}\right)^{1 / 2}
\end{aligned}
$$

and

$$
\begin{aligned}
g_{6 / 11}= & \frac{2^{7 / 4}}{(3+\sqrt{33}-\sqrt{26+6 \sqrt{33}})^{1 / 12}(\sqrt{2}+\sqrt{3})^{1 / 4}(7 \sqrt{2}+3 \sqrt{11})^{1 / 12}} \\
& \times \frac{1}{(\sqrt{7+\sqrt{33}}+\sqrt{\sqrt{33}-1})^{1 / 2}} .
\end{aligned}
$$

Proof. As in the previous proofs, we express Lemma 2.8 in eta-function $f$, replace $q$ by $-q$ and then use the definition of $r_{2, n}$, set $n=1 / 33$ and employ(1.8) to deduce that

$$
x^{4}+\frac{1}{x^{4}}-3\left(x^{2}+\frac{1}{x^{2}}\right)-4=0,
$$

where $x=\left(r_{2,11 / 3} / r_{2,33}\right)$.

Solving the (3.38) for $x$ and considering that $r_{2, n}>1$ for $n>1$ and applying (1.7), we obtain

$$
x:=\left(\frac{r_{2,11 / 3}}{r_{2,33}}\right)=\left(\frac{g_{22 / 3}}{g_{66}}\right)=\frac{1}{2}(3+\sqrt{33}-\sqrt{26+6 \sqrt{33}})^{1 / 2} .
$$

Again, from [5, p. 201], we note that

$$
g_{66}=(\sqrt{2}+\sqrt{3})^{1 / 4}(7 \sqrt{2}+3 \sqrt{11})^{1 / 12}\left(\sqrt{\frac{7+\sqrt{33}}{8}}+\sqrt{\frac{\sqrt{33}-1}{8}}\right)^{1 / 2}
$$

Combining (3.40) and (3.39), we arrive at the value of $g_{22 / 3}$. Similarly, applying (1.7) and (1.8), the value of $g_{6 / 11}$ can be obtained from (3.39) and (3.40). 
Theorem 3.10. We have

$$
g_{6 / 13}=\left(\frac{-3+\sqrt{13}}{2}\right)^{1 / 2}(5+\sqrt{26})^{1 / 6}
$$

and

$$
g_{26 / 3}=\frac{2}{(5+\sqrt{26})^{1 / 6} \sqrt{-6+2 \sqrt{13}}} .
$$

Proof. By routine work, applying the definition of $r_{2, n}$ in Lemma 2.7, setting $n=1 / 39$ and using (1.8), we obtain

$$
\left(\frac{r_{2,3 / 13}}{r_{2,39}}\right)^{2}+\left(\frac{r_{2,3 / 13}}{r_{2,39}}\right)^{-2}-11=0 .
$$

Solving the $(3.41)$ for $\left(r_{2,3 / 13} / r_{2,39}\right)$ and noting that $r_{2, n}>1$ for $n>1$ and $g_{n}=r_{2, n / 2}$, we find that

$$
\left(\frac{r_{2,3 / 13}}{r_{2,39}}\right)=\left(\frac{g_{6 / 13}}{g_{78}}\right)=\left(\frac{11-3 \sqrt{13}}{2}\right)^{1 / 2} .
$$

Now, from [5, p. 202], we have

$$
g_{78}=\left(\frac{3+\sqrt{13}}{2}\right)^{1 / 2}(5+\sqrt{26})^{1 / 6} .
$$

Combining (3.42) and (3.43), we obtain the value of $g_{6 / 13}$. In a similar way, employing (1.7) and (1.8), we arrive at the value of $g_{26 / 3}$.

\section{References}

[1] N.D. Baruah, On some of class invariants of Ramanujan, J. Indian Math. Soc. 68(1-4) (2001), 113-131.

[2] N.D. Baruah, On some of Ramanujan's Schläfli-type " mixed "modular equations, J. Number Theory 100 (2003), 270-294.

[3] B.C. Berndt, Ramanujan's Notebooks, Part III, Springer-Verlag, New York, 1991.

[4] B.C. Berndt, Ramanujan's Notebooks, Part IV, Springer-Verlag, New York, 1994.

[5] B.C. Berndt, Ramanujan's Notebooks, Part V, Springer-Verlag, New York, 1998.

[6] S. Ramanujan, Modular equations and approximations to $\pi$, Quart. J. Math. 45 (1914), 350-372.

[7] S. Ramanujan, Notebooks (2 volumes), Tata Institute of Fundamental Research, Bombay, 1957. 
[8] N. Saikia, Ramanujan's modular equations and Weber-Ramanujan's class invariants $G_{n}$ and $g_{n}$, Bull. Math. Sci. 2 (2012), 205-223.

[9] N. Saikia, A Parameter for Ramanujan's Function $\chi(q)$ : Its Explicit Values and Applications, ISRN Comput. Math., vol. 2012 (2012), article ID 169050, doi: $10.5402 / 2012 / 169050$.

[10] J. Yi, Construction and application of modular equation, Ph. D thesis, University of Illionis, 2001.

[11] J. Yi, Theta-function identities and the explicit formulas for theta-function and their applications, J. Math. Anal. Appl. 292 (2004), 381-400.

Address: Nipen Saikia: Department of Mathematics, Rajiv Gandhi University, Rono Hills, Doimukh-791112, Arunachal Pradesh, India.

E-mail: nipennak@yahoo.com

Received: 14 February 2012; revised: 5 August 2013 
